\title{
Dielectric Relaxation of Poly(n-butyl acrylate)
}

\author{
Tadashi HaYaKaWA ${ }^{\dagger}$ and Keiichiro ADACHI ${ }^{\dagger \dagger}$ \\ Department of Macromolecular Science, Graduate School of Science, \\ Osaka University, Toyonaka, Osaka 560-0043, Japan
}

(Received March 9, 2000; Accepted June 26, 2000)

\begin{abstract}
Dielectric relaxation of poly(n-butyl acrylate) (PBA) was studied over a temperature range from 90 to $300 \mathrm{~K}$. The primary $\alpha$ relaxation was observed around $250 \mathrm{~K}$ but the relaxation due to motion of the side ester group as observed for poly (methyl acrylate) ( $\beta$ process) was not observed. Thus the $\alpha$ relaxation was assigned to a merged process where the segmental motions and the rotation of the side group occur cooperatively. A weak secondary $(\gamma)$ relaxation was observed at $130 \mathrm{~K}$ at $1 \mathrm{kHz}$. The $\gamma$ relaxation was assigned to motion of the alkyl group of the side chain. Dielectric characteristics such as relaxation strength, effective dipole moment, and Arrhenius plot for the $\alpha$ process were compared with those for poly(butyl methacrylate)s reported.

KEY WORDS Poly $(n$-butyl acrylate) / Dielectric Relaxation / $\alpha$ Relaxation / $\gamma$ Relaxation / Effective Dipole Moment/
\end{abstract}

Dielectric properties of poly(alkyl methacrylate) (PAMA) and those of poly(alkyl acrylate) (PAA) are much different. ${ }^{1-5}$ If a PAMA is compared with the corresponding PAA having the same alkyl group, the former exhibits higher glass transition temperature $T_{\mathrm{g}}$ than the latter. ${ }^{1}$ The dielectric behavior of PAMA is sensitive to stereoregularity but PAA is not. ${ }^{1}$ The differences between PAMA and PAA are believed due to differences in the barrier height for the internal rotation of the backbone chains. Poly(methyl methacrylate) (PMMA) exhibits a strong $\beta$ relaxation due to rotation of the side group but poly(methyl acrylate) (PMA) exhibits a moderate $\beta$ relaxation. ${ }^{1,2,5}$ In PAMAs having the ethyl, propyl and higher alkyl groups, the $\alpha$ and $\beta$ relaxations merge and the $\beta$ relaxation due to rotation of the side ester group cannot be observed separately. ${ }^{1-4}$ Ishida and Yamafuji reported dielectric data on PAMA and PAA ca. 40 years ago and attempted to explain the behavior using a site model. ${ }^{2}$ They explained the dielectric behavior of PAMA qualitatively based on their model. Currently, however, many scientists consider that cooperative intermolecular interactions play important role in segmental motion of bulk polymers. ${ }^{6-9}$ Therefore further analysis based on the intra and intermolecular interactions is needed for interpretation of differences between PAMA and PAA. To discuss differences in the dielectric behavior between PAMA and PAA, we need to compare the dielectric behavior of these homologues having the same side group. Several authors reported the dielectric behavior of poly ( $n$-butyl methacrylate) (PBMA), ${ }^{2-4}$ but, as far as we know the dielectric properties of poly(n-butyl acrylate) PBA have not been reported. This paper reports the dielectric properties of PBA focusing our attention on the above. Due to intermolecular cooperative effects, temperature dependence of relaxation time for the $\alpha$ process of flexible polymers conforms to the Vogel-Fulcher equation. ${ }^{10,11}$ The cooperative effects should cause anti-parallel or parallel orientation of the neighboring dipoles depending on the in- termolecular interactions and are reflected on the Kirkwood dipolar correlation factor for the $\alpha$ process. ${ }^{12,13}$ Our objective is to compare the dielectric behavior for PBA and PBMA.

\section{EXPERIMENTAL}

\section{Material}

PBA was purchased from Aldrich Chemical Company Inc. The sample had very broad distribution of molecular weight. Therefore we carried out fractionation by adding methanol to 1 to $0.1 \mathrm{wt} \%$ tetrahydrofuran (THF) solutions. A fraction of high molecular weight was collected. Characterization of the sample was made by GPC equipped with a low angle light scattering detector (Tosoh LS 8000). The weight average molecular weight $M_{\mathrm{w}}$ was $3.80 \times 10^{5}$ and the polydispersity index $M_{\mathrm{w}} / M_{\mathrm{n}}$ was 1.38 .

\section{Method}

Dielectric measurements were carried out with an RLC bridge (QuadTech Type 1693, Ohio, USA) in the range from $12 \mathrm{~Hz}$ to $200 \mathrm{kHz}$. A dielectric cell designed for measurement of viscous polymer samples was reported previously. ${ }^{14}$

\section{RESULTS AND DISCUSSION}

\section{Dielectric Behavior of PBA}

Figures $1 \mathrm{a}$ and $1 \mathrm{~b}$ show the temperature dependence of the dielectric constant $\varepsilon^{\prime}$ and loss factor $\varepsilon^{\prime \prime}$ of PBA, respectively. Strong loss peaks are seen in the range from 230 to $270 \mathrm{~K}$. The glass transition temperature $T_{\mathrm{g}}$ was determined by means of differential scanning calorimetry (DSC) as $218 \mathrm{~K}$ at the heating rate of $5 \mathrm{~K} \mathrm{~min}^{-1} .^{15}$ Therefore the dielectric loss peak observed in the range 230 to $270 \mathrm{~K}$ can be assigned to the primary $\alpha$ process. A small secondary $(\gamma)$ relaxation appears around $140 \mathrm{~K}$. The peak height of $\varepsilon^{\prime \prime}$ for the $\gamma$ relaxation is only 0.03 ,

\footnotetext{
${ }^{\dagger}$ Present address: Nippon NSC Co., 1-6-5 Senba-nishi, Minou, Osaka 562-8586, Japan.

${ }^{\dagger+}$ To whom correspondence should be addressed.
} 

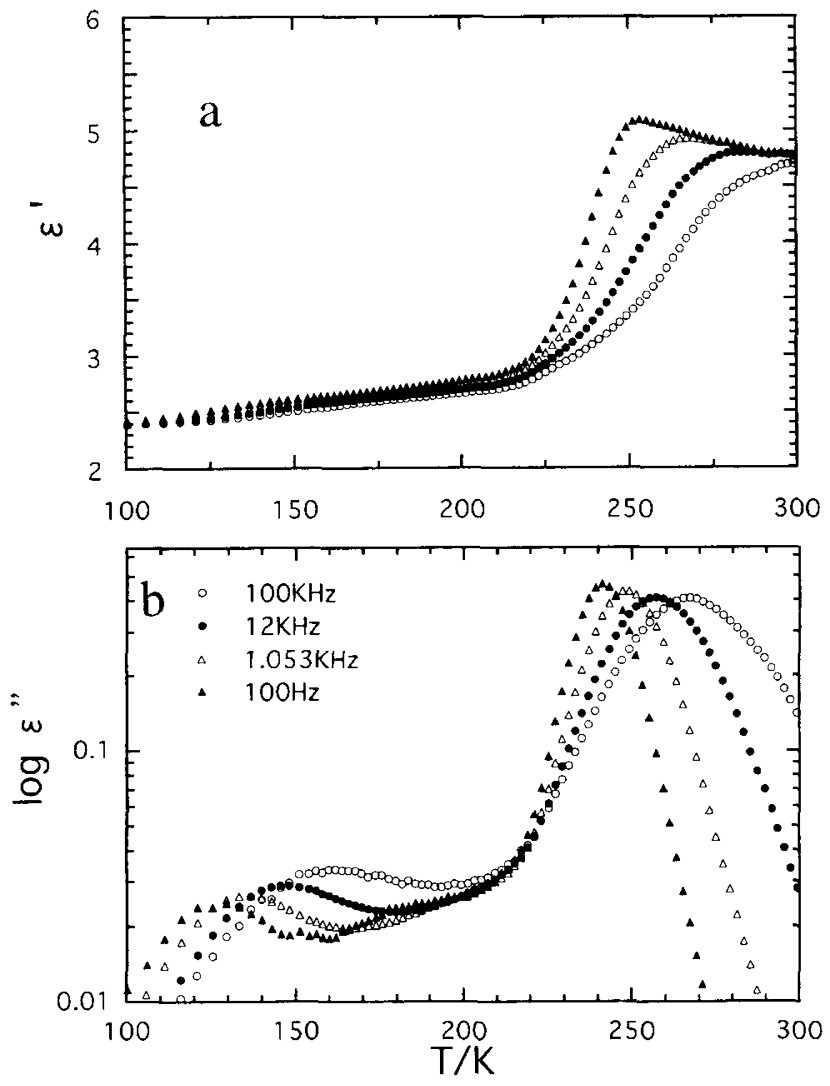

Figure 1. Temperature dependence of the dielectric constant $\varepsilon$ ' (a) and logarithm of the loss factor $\varepsilon^{\prime \prime}(\mathrm{b})$.
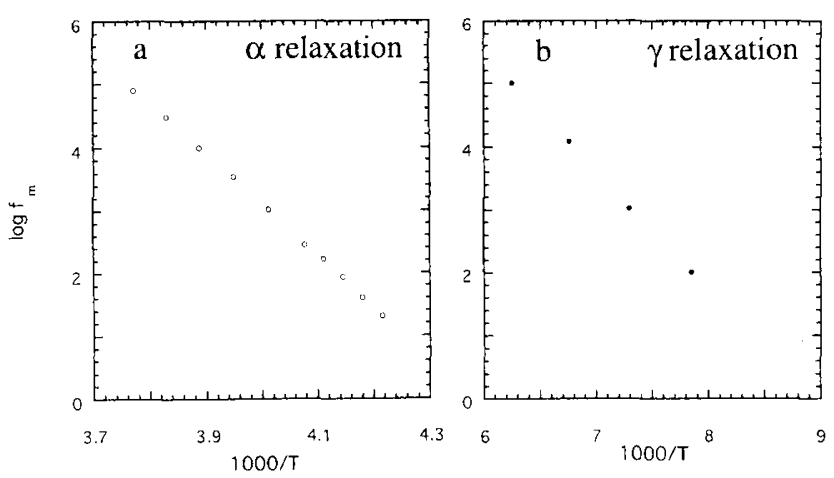

Figure 2. Arrhenius plots of the $\alpha$ and $\gamma$ processes.

much smaller than the $\beta$ relaxations of PMMA or PMA assigned to the rotation of the ester group. ${ }^{1,2,5}$ Thus the $\gamma$ relaxation of PBA cannot be assigned to overall rotation of the side group. To avoid confusion, we designate the present secondary process as $\gamma$ relaxation.

The Arrhenius plots of the loss maximum frequencies $f_{\mathrm{m}}$ for the $\alpha$ and $\gamma$ relaxations are shown in Figure 2. To our surprise the Arrhenius plot for the $\alpha$ process almost conforms to a straight line unlike many amorphous polymers. However the plot is slightly curved. We attempted to fit the plot to the Vogel-Fulcher equation: ${ }^{10,11}$

$$
\log f_{\mathrm{m}}=A-B /\left(T-T_{\mathrm{o}}\right)
$$

where $A, B$, and $T_{0}$ are adjustable parameters deter-

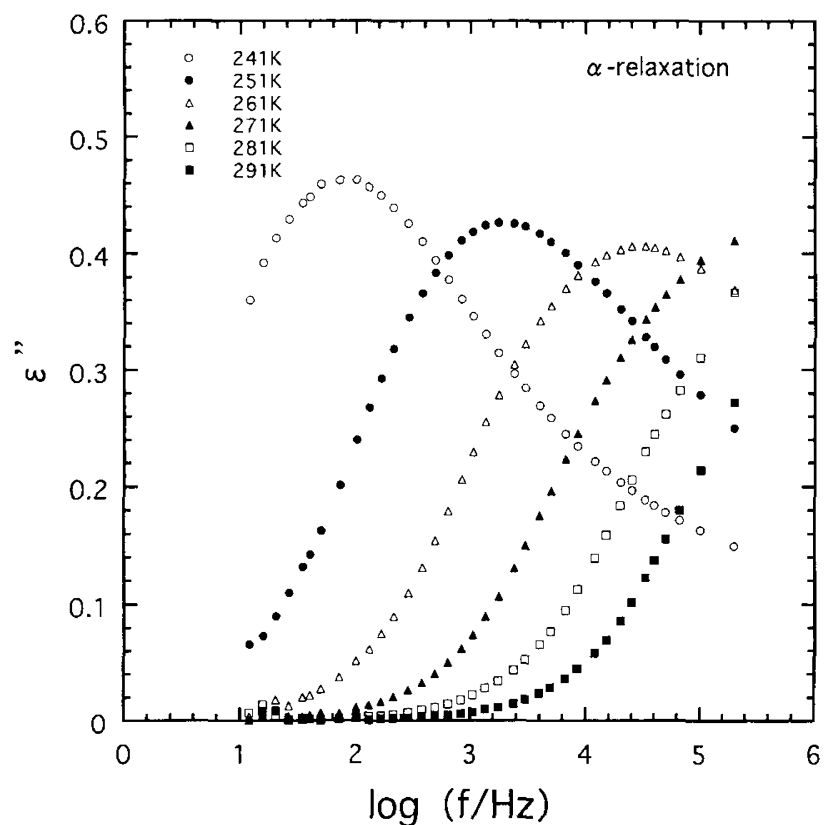

Figure 3. Frequency dependence of the dielectric loss factor $\varepsilon$ ".

mined as $37.0,4560$, and 102 , respectively. $A$ for many polymers is 12 to 14 , while for the present polymer $\mathrm{PBA}$, $A$ is higher than the ordinary value. Furthermore $B$ of PBA is unusually high. From the Arrhenius plot of PBMA reported by Ishida and Yamafuji, ${ }^{2}$ we obtained $A$ $=18.5, B=1620$, and $T_{0}=174.5$ for PBMA. Although $A$ and $B$ for PBMA are smaller than those of PBA, $A$ and $B$ of PBMA are still higher than ordinary values. The nonlinear Arrhenius plot is a manifestation of the cooperative motion of polymer segments. ${ }^{6-9}$ The long butyl side group may hinder cooperative motion.

The Arrhenius plot for the $\gamma$ process is straight. The activation energy for the $\gamma$ process is $30.8 \mathrm{~kJ} \mathrm{~mol}^{-1}$.

\section{$\alpha$ Relaxation}

Figure 3 shows the frequency dependence of $\varepsilon$ " at temperatures where the $\alpha$ process is seen. The height of the loss peak increases with decreasing temperature as observed for ordinary polymers. These $\varepsilon^{\prime \prime}$ curves are approximately superposable. This behavior is much different from the behavior in the $\alpha$ process of poly ( $n$-butyl methacrylate) (PBMA) in which the peak height decreases with decreasing temperature and the $\varepsilon$ " curve broadens remarkably with decreasing temperature. ${ }^{2}$ The $\varepsilon^{\prime \prime}$ curves for the present PBA were cast to the HavriliakNegami equation: ${ }^{16}$

$$
\varepsilon^{\prime}-i \varepsilon^{\prime \prime}=\frac{\Delta \varepsilon}{\left[1+\left(i \omega \tau_{0}\right)^{\alpha}\right]^{\beta}}
$$

where $\alpha$ and $\beta$ are parameters determined as 0.57 and 0.36 , respectively. For typical amorphous polymers, $\alpha$ and $\beta$ are 0.8 and 0.5 , respectively. ${ }^{16}$ The parameters for PBA are relatively small compared with ordinary amorphous polymers such as poly(vinyl acetate) indicating segmental dynamics for polymers having a long flexible side group to differ from polymers having relatively short side groups. 

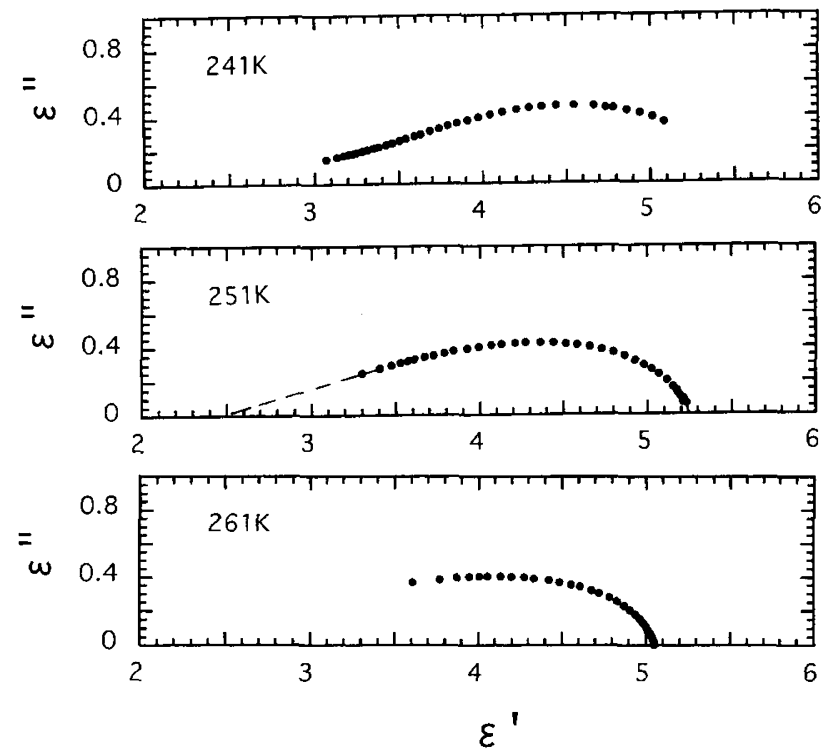

Figure 4. Cole-Cole plots of the $\alpha$ process.

Figure 4 shows representative Cole-Cole diagrams for the $\alpha$ relaxation. From the data at $251 \mathrm{~K}$, the relaxation strength $\Delta \varepsilon$ was determined to be 2.85 . According to Onsager $^{17}$ and Kirkwood ${ }^{12}, \Delta \varepsilon$ is given by (in cgs unit),

$$
\Delta \varepsilon=\frac{4 \pi N g \mu^{2}}{9 k_{\mathrm{B}} T} \frac{\varepsilon_{\mathrm{R}}\left(\varepsilon_{\mathrm{U}}+2\right)^{2}}{2 \varepsilon_{\mathrm{R}}+\varepsilon_{\mathrm{U}}}
$$

where $N$ is the number of the monomeric units in unit volume; $g$, the dipolar correlation factor, $\varepsilon_{\mathrm{R}}$, the relaxed dielectric constant; and $\varepsilon_{\mathrm{U}}$, the unrelaxed dielectric constant. $\varepsilon_{\mathrm{R}}$ and $\varepsilon_{\mathrm{U}}$ are given by the Cole-Cole diagram and $N$ is calculated from the density of PBA given by Aldrich Co. as 1.087. From these data $g \mu^{2}\left(=\mu_{\text {eff }}{ }^{2}\right)$ of eq 3 is calculated as $1.952 \times 10^{-59} \mathrm{C}^{2} \mathrm{~m}^{2}\left(=1.755 \times 10^{-36} \mathrm{cgs}\right.$ esu $)$ and the effective dipole moment $\mu_{\text {eff }}$ to be $4.40 \times 10^{-30}$ $\mathrm{C} \mathrm{m}\left(=1.32 \times 10^{-18} \mathrm{cgs}\right.$ esu=1.32 D). If the dipole moment $\mu$ of the monomeric unit is taken to be $1.80 \mathrm{D},{ }^{18} \mathrm{~g}$ becomes 0.54 . This indicates that the dipoles of the monomer units tend to orient in the antiparallel direction. For poly(butyl methacrylate) (PBMA) we calculated $\mu_{\text {eff }}$ to be $1.30 \mathrm{D}$ from the data at $333 \mathrm{~K}$ reported by Ishida and Yamafuji. ${ }^{2}$ This value is very close to $\mu_{\text {eff }}$ of PBA. Therefore, the dipolar correlations of PBA and PBMA are the same.

\section{$\gamma$ Relaxation}

The $\varepsilon$ " $v s$. frequency curve for the $\gamma$ region was very broad and hence the Cole-Cole plots could not be used for determination of the relaxation strength $\Delta \varepsilon$. To determine $\Delta \varepsilon$, we used the $\varepsilon^{\prime \prime} v s .1 / T$ plots: ${ }^{1}$

$$
\Delta \varepsilon=\left(\frac{2 E_{\mathrm{a}}}{\pi R}\right) \int_{0}^{\infty} \varepsilon^{\prime \prime} \mathrm{d}\left(\frac{1}{T}\right)
$$

where $E_{\mathrm{a}}$ is the activation energy. Figure 5 shows the $\varepsilon$ " vs. $1 / T$ plots in the $\gamma$ region. The curve at $100 \mathrm{~Hz}$ is extrapolated as indicated by the dashed line and from the area of the curve, $\Delta \varepsilon$ is determined to be 0.19 . From the Onsager equation, ${ }^{17}$ the effective dipole moment for the $\gamma$ relaxation process was determined to be $0.28 \mathrm{D}$.

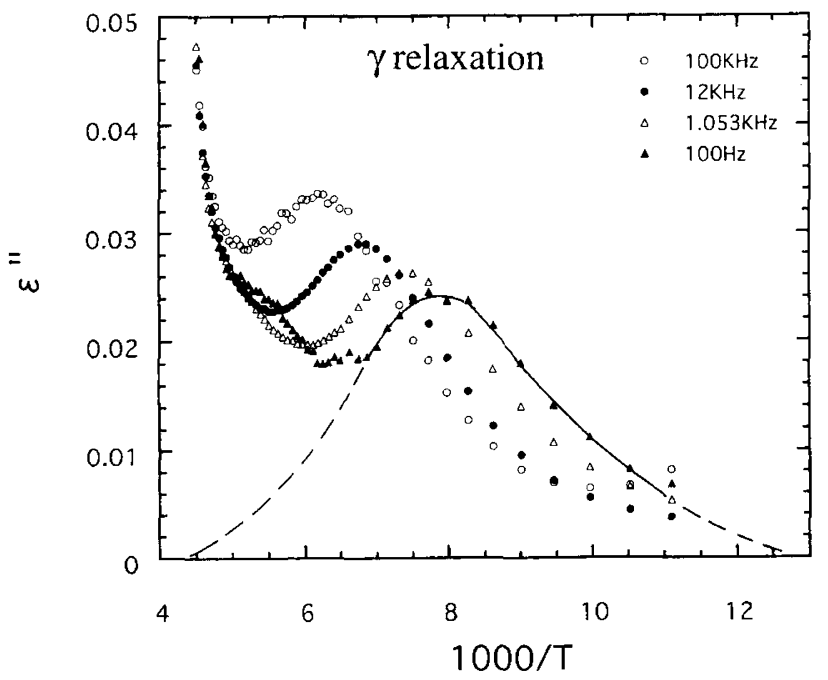

Figure 5. Plots of $\varepsilon^{\prime \prime} v s .1 / T$ in the $\gamma$ relaxation region. Dashed line indicates an estimated curve used to determine the relaxation strength.

The side group has many bonds and thus various degrees of freedom of internal rotation. Hoff et al. reported the mechanical measurements on PBMA, ${ }^{18}$ and found a weak relaxation around $100 \mathrm{~K}$. They assigned the relaxation to the motion of the butyl groups. Unfortunately, no dielectric data of PBMA in the low temperature range are available. Here we test this possibility for the $\gamma$ relaxation. To discuss motions of the side group, we label the atoms of the side chain as, $-\mathrm{C}_{1} \mathrm{O}-\mathrm{O}_{1}-\mathrm{C}_{2}-\mathrm{C}_{3}$ $-\mathrm{C}_{4}-\mathrm{C}_{5}$.

Since the dipole moments of normal alkanes are zero, the rotation around the bonds $\mathrm{C}_{2}-\mathrm{C}_{3}$ or $\mathrm{C}_{3}-\mathrm{C}_{4}$ is not active in dielectric spectra. Then we calculated the barrier heights for internal rotation around the $\mathrm{O}_{1}-\mathrm{C}_{2}$ bond based on the MM2 force field. ${ }^{19}$ The potential minimum arises at \pm 80 (gauche) and $180^{\circ}$ (trans). The difference of the dipole moments for the trans and gauche states was $0.27 \mathrm{D}$. Although the reliability of this value must be checked, it agrees with the observed effective dipole moment. The barrier height between the gauche and trans sites is $4.6 \mathrm{~kJ} \mathrm{~mol}^{-1}$ and between the two gauche sites, $12 \mathrm{~kJ} \mathrm{~mol}^{-1}$. These barrier heights are much lower than the observed activation energy $\left(30.8 \mathrm{~kJ} \mathrm{~mol}^{-1}\right)$ for the $\gamma$ process. The discrepancy may be attributed to intermolecular interactions. Thus we may assign the $\gamma$ relaxation to the motion of the butyl group.

\section{CONCLUSION}

Dielectric behavior of poly( $n$-butyl acrylate) was examined. Two relaxations $\alpha$ and $\gamma$ were observed at 250 and $140 \mathrm{~K}$, respectively. The $\alpha$ relaxation can be assigned to a process in which the segmental motion and the rotation of the ester group $(\beta)$ occur cooperatively. The parameters $A$ and $B$ of the Vogel-Fulcher equation are higher than those of ordinary polymers. The dipolar correlation factor of 0.5 is similar to that of PBMA. The $\gamma$ relaxation can be assigned to motion of the butyl group around the $\mathrm{C}-\mathrm{O}$ bond of the ester group. 


\section{REFERENCES}

1. N. G. McCrum, B. E. Read, and G. Williams, "Anelastic and Dielectric Effects in Polymeric Solids", John Wiley \& Sons, New York, N.Y., 1967, Chapter 8, pp 238-299 and references cited there (Reprinted by Dover Publ. Inc. New York, N.Y., 1991).

2. Y. Ishida and K. Yamafuji, Kolloid Z., 177, 7 (1961).

3. L. de Brouckere and G. Offergeld, J. Polym. Sci., 30, 105 (1958).

4. J. D. Ferry and S. Strella, J. Colloid Sci., 13, 459 (1958).

5. Y. Ishida, Kolloid Z., 174, 162 (1961).

6. G. Adam and J. H. Gibbs, J. Chem. Phys., 43, 139 (1965).

7. K. Adachi, Macromolecules, 23, 1816 (1990).

8. S. Matsuoka and X. Quan, Macromolecules, 24, 2770 (1991).

9. K. L. Ngai, A. K. Pajagopal, and S. Teitler, J. Chem. Phys. 88,
6088 (1988).

10. H. Vogel, Physik. Z., 22, 645 (1921).

11. G. A. Fulcher, J. Am. Ceram. Soc., 8, 339 (1925).

12. J. G. Kirkwood, J. Chem. Phys., 7, 911 (1939).

13. R. Diaz-Calleza and E. Riande, "Dielectric Spectroscopy of Polymeric Materials", J. P. Runt and J. J. Fitzgerald, Ed., American Chemical Society, Washington, D. C., Chapter 5, pp 139-173, 1997.

14. Y. Imanishi, K. Adachi, and T. Kotka, J. Chem. Phys., 89, 7585 (1988).

15. T. Hayakawa and K. Adachi, Macromolecules, in press.

16. S. Havriliak and S. Negami, Polymer, 8, 161 (1967).

17. L. Onsager, J. Am. Chem. Soc., 58, 1486 (1936).

18. E. A. W. Hoff, D. W. Robison, and A. H. Willbourn, J. Polym. Sci. 18, 161 (1955).

19. U. Burkert and N. L. Allinger "Molecular Mechanics", American Chemical Society, Washington, D.C., MD 1982. 